\title{
Combining Inertial Measurements and Distributed Magnetometry for Motion Estimation
}

\author{
Eric Dorveaux, Thomas Boudot, Mathieu Hillion, Nicolas Petit
}

\begin{abstract}
We address the problem of estimating the position of a rigid body moving indoors. Disturbances of the magnetic field observed in buildings are used to derive a reliable velocity estimate. The estimated velocity is expressed in the body reference frame, which imposes to simultaneously reconstruct the rotation of this frame with respect to an inertial frame of reference. For this, an inertial measurement unit (IMU) is used. To maximize the accuracy of the reconstructed motion, alignment and calibration of the inertial sensors have to be carefully treated, which minimizes projection errors. A first contribution of this paper is an alignment-calibration technique combining gyrometers and accelerometers to address the attitude estimation problem. A second contribution is an observer of the velocity, the convergence of which is proved. Finally, an experimental testbench is described and experimental results are provided.
\end{abstract}

\section{INTRODUCTION}

Lately, the demand for indoor positioning and navigation technology has been growing, as popular handheld devices and smartphones, which have numerous sensors and computational capabilities, have reached mass market with an immense success. Many different techniques have been investigated. However, none is capable of covering the whole range of possible applications. Some approaches relying on pre-installed infrastructures (e.g. radio emitters like UWB, WLAN or RFID beacons) have shown very good results, provided preliminary installation of equipments can be performed. However, this assumption cannot always hold. In numerous application (e.g. buildings on fire), first responders may have to intervene in any type of buildings whether it is equipped with such a system or not. Moreover, the conditions of their interventions may often prevent or at least modify the use of certain positioning methods. The use of traditional techniques based on computer vision is totally discarded in the case of smoky underground tunnels or parking lots for instance.

In the most general case where the conditions of intervention, and the availability of pre-installed equipments are unknown, inertial systems (see e.g. [4], [8]) represent a dependable and robust technology as they keep working. An Inertial Measurement Unit (IMU) delivers accelerometers,

\footnotetext{
E. Dorveaux (corresponding author) is a $\mathrm{PhD}$ candidate in Mathematics and Control, CAS, Unité Mathématiques et Systèmes, Mines-ParisTech, 60, bd St Michel, 75272 Paris, France eric.dorveaux@mines-paristech. fr

T. Boudot is a graduate student at Ecole Polytechnique, Route de Saclay, 91128 Palaiseau, France

M. Hillion is with SYSNAV, Zone industrielle B, 1 rue Jean de Becker Rémy, BP86, 27940 Aubevoye, France

N. Petit is with CAS, Unité Mathématiques et Systèmes, Mines-ParisTech, 60, bd St Michel, 75272 Paris, France
}

gyroscopes (and most often magnetometers) signals which can be used to derive a position information through a double integration process (see e.g. [8], [10]). However, because of the sensors drifts, this approach requires very high precision IMUs, such as those found in certain full-sized aircrafts. When cost, space, and weight constraints become stringent, other information has to be used to reduce or eliminate the drifts. The availability of maps, for instance, can allow to periodically eliminate the drifts, whenever a precise location is recognized (steps, lifts, corners, turns... See e.g. [18] for some simple experimental results). Many works have been conducted on the particular case where the IMU is mounted on the foot (see e.g. [13]). Velocities update techniques (ZUPTs) (see [15] for a comparison of various ZUPT detectors) allow to reduce the integration to small steps between phases where the foot is at rest on the ground. The drift is thus much lowered, especially for the accelerometer, which allows a longer use before diverging too far away from the actual position. Nevertheless, as first responders are concerned, feet are not the best place to put sensors on: it is often the first part of the body that hits a wall, a door or any piece of furniture that is in the way.

An other approach to improve the efficiency of inertial systems as been first proposed in [17], [16]. It relies on a set of magnetometers to monitor the magnetic field and its spatial derivatives. A velocity information, which can allow to reduce greatly the drifts of an inertial navigation technique, can be derived from these measurements. The focus of this article is to expose important steps in the development of such a distributed magnetometry-based approach to estimate the velocity and, eventually, the motion of a rigid body in a magnetically disturbed environment. A velocity estimate relying solely on magnetometers (used to estimate the velocity in the body frame) and on accelerometers and gyrometers (used to estimate the attitude of the whole system, and thus the velocity in the inertial frame) is proposed. The performance of such a system is closely related to the sensors calibration. This is the first problem we address.

First, the numerous magnetometers that are used must be jointly calibrated to cancel biases and scale factors of the sensing axes. This task, which was addressed in [5] and [6], is of paramount importance to enable the sensors to deliver sensible information on the velocity of the system in the body frame of reference. Secondly, the IMU (accelerometer and gyrometer), which is used to convert the obtained velocity estimate from the body frame to the inertial frame, has to be calibrated as well. As will be discussed in details, it is desirable to propose simple, yet effective, calibration 
methods that can be used in-the-field, i.e. without requiring heavy, expensive or cumbersome calibration equipments.

The article is organized as follows. In Section I, the model of the magnetic field and of the rigid body dynamics are presented along with the notations used throughout the paper. In Section II, the calibration of the sensors used is described. The emphasis is on the calibration of the gyroscope with respect to the accelerometers. Then, an observer is proposed in Section III to estimate the magnetic field, and most importantly, the velocity in the body frame. A proof of convergence of this observer to the actual magnetic field and the actual velocity is given. Finally, in Section IV an experimental testbench is presented and some results stressing the relevance of the approach are provided.

\section{PRoblem Statement}

\section{A. Notations}

We consider a rigid body with strapdown (i.e. attached to the rigid body) magnetometers and inertial sensors in view of estimating its motion. Two frames of coordinates are used to represent the problem:

- a local inertial frame of reference: $\Re_{i}$

- a body frame: $\Re_{b}$

Denote $\mathrm{R}$ the rotation matrix between those two frames, from the inertial reference frame $\Re_{i}$ to the body frame $\Re_{b}$, and $\Omega=\Omega_{b / i}$ the instantaneous rotation vector of the body frame with reference to the inertial frame.

The rigid body under consideration can simultaneously translate and rotate in 3D. We desire to estimate the velocity of this platform by using solely measurements of the magnetic field and of the rate of turn.

Variables expressed in the inertial reference frame are marked by the subscript $i$. In details, we consider the following variables:

- $H_{i}$ the magnetic field (three dimensional vector)

- $v_{i}$ the speed of the rigid body (three dimensional vector)

- $J_{i}(H)$ the (3x3 matrix) Jacobian of the magnetic field, defined by

$$
J_{i}(H)=\left(\frac{\partial H_{k}}{\partial x_{l}}\right)_{(k, l)}
$$

When those quantities are expressed in the body frame, subscript $b$ is used. By definition, we have

$$
\left.\begin{array}{rl}
H_{b} & =R H_{i} \\
v_{b} & =R v_{i} \\
J_{b}(H) & =R J_{i}(H) R^{T}
\end{array}\right\}
$$

\section{B. Equations of motion}

The most common way to use magnetic measurements in attitude and position estimation techniques is to consider they give a direct reading of the heading vector. This approach gives very good results provided that magnetic disturbances are small. However, this is not the case indoor where the heading information can vary by more than 30 degrees during a simple translation of a few meters (see [16] for experimental results stressing this point). As already shown in [17], those disturbances can be related to the velocity assuming that the magnetic field depends only on space and not on time, i.e. assuming stationarity of this variable. This relation is obtained, in the inertial frame $\Re_{i}$, by the chain rule

$$
\dot{H}_{i}=J_{i}(H) v_{i}
$$

As measurements of the magnetic field and its Jacobian are naturally performed in the body frame $\Re_{b}$, the relation is more useful expressed in that frame (see Appendix II for a detailed derivation of this relation)

$$
\dot{H}_{b}=-\Omega \times H_{b}+J_{b}(H) v_{b}
$$

In the general case, the motion of a rigid body is expressed in the body frame by Newton's law

$$
\dot{v_{b}}=-\Omega \times v_{b}+F_{m}
$$

where $F_{m}$ is the total force per unit of mass applied to the rigid body. Assuming that the pedestrian considered as a rigid body keeps moving ahead during turns, one can model these forces as

$$
F_{m}=\Omega \times v_{b}
$$

so that

$$
\dot{v_{b}}=0
$$

Finally, gathering (4) and (7), the dynamics of our system is described in the body frame by

$$
\left\{\begin{aligned}
\dot{H}_{b} & =-\Omega \times H_{b}+J_{b}(H) v_{b} \\
\dot{v}_{b} & =0
\end{aligned}\right.
$$

In practice, a gyroscope provides a measure of the rate of turn $\Omega$ of the moving body with respect to the reference frame $\Re_{i}$.

A set of spatially distributed magnetometers delivers two measurements. First, this set gives the value of the magnetic field in the body frame $H_{b}$ by a direct reading. Secondly, by making use of the data provided by all sensors of the set, the Jacobian of the magnetic field in the body frame $J_{b}(H)$ can be estimated by a finite difference scheme. A simple 2Dexample is presented in Section IV-A. Computing differences between the sensors requires to take great care over their calibration since the difference between the readings is much lower than the sensed field. As the only information regarding the velocity is given through these computed spatial differences between the magnetometers, its accuracy is of high importance. We address this problem in the following section. Eventually, an estimate of the matrix $R$ is needed to reconstruct the velocity, and thus the motion, in the inertial frame of reference $\Re_{i}$. For this task, accelerometers and gyrometers can be used to obtain an attitude observer (see e.g. [11]).

\section{Prerequisite: Sensors CAlibration}

As discussed in Section I, several sensors are required (a set of magnetometers, an accelerometer and a gyrometer) to estimate the attitude and the velocity of the rigid body under consideration. To allow them to work together, a calibration 
step has to be performed. The main errors usually accounted for are the following ones:

- Errors in the sensor frame: among these are biases, scale factors, orthogonality errors and cross-coupling between the three sensing axes.

Practically, only the first order of these terms is sought after, according to a model of the form

$$
F_{\text {true }}^{\text {sensor }}=A F_{\text {measure }}^{\text {sensor }}+b
$$

where $F_{\text {true }}^{\text {sensor }}$ is the true value of the sensed field $F$ we want to compute in the sensor frame, $F_{\text {measure }}^{\text {sensor }}$ is the corresponding measured value of the same field in the sensor frame, $A$ is the calibration matrix accounting for scale factors, orthogonality errors, cross-coupling, and $b$ is a bias vector. $A$ and $b$ are the parameters to be estimated during the calibration procedure.

- Harmonization errors between the various sensor frames. Typically, rotations up to 3 degrees are generally observed between two sensor frames.

In this section, a calibration along with an harmonization procedure are presented for the set of magnetometer. Secondly, calibration of accelerometer and gyrometer along with their harmonization is provided.

\section{A. Magnetometers calibration}

The iterative algorithm proposed in [5] gives the calibration matrices as the result of a sequence of least square problems in which the input data are iteratively calibrated. We do not recall this algorithm, details on its properties (convergence and efficiency) can be found in [5]. In summary, the least-square problems are used to seek calibration parameters such that the sense field (in practice the Earth magnetic field) has a constant norm when the sensors are rotated in every possible direction. Further, in [7], some strategies to harmonize (i.e. perform an alignment of) a whole set of magnetometers in the same frame are presented, either by first correcting the flaws of each sensor and then performing an harmonization step which often gives a particular roll to one sensor, or by getting rid of the two kinds of errors simultaneously. This later algorithm is used to set all the magnetometers in one frame.

\section{B. Gyrometer and accelerometer calibration}

Classically (see [11], and the references therein), the attitude of a rigid body (described by a rotation matrix for instance) can be estimated using accelerometers and gyrometers. The attitude is used to project the velocity from the body frame into the inertial frame of reference. Integration of the inertial frame velocity provides the trajectory. Since small angular errors in the attitude estimation give linearly growing errors in the trajectory, the attitude estimation is also a key task of our system. Calibration and harmonization of the accelerometers and gyrometers is then of paramount importance.

The accelerometer, which measures (at rest) the Earth gravity field (which is by assumption of constant norm in a neighborhood of the rigid body), can be calibrated using the exact same technique as the one presented for magnetometers. Bias and scale factors are then identified and removed. However, this method is not suitable for gyroscope calibration. The culprit is that having some precisely controlled rotation rate is very difficult during in-the-field experiments. This results in a sensed field that does not have a constant norm, which totally discards the previously presented methods. The method presented below uses the relation between the accelerometer measurements and the rate of turn, as given by the chain rule differentiation. Compared to other methods (as e.g. [9]), the dynamic is not integrated, and the equation are kept under their differential expressions. The result of this calibration procedure is that the gyrometer is calibrated and harmonized with the accelerometer.

Let us define the calibration problem we wish to address.

\section{Problem Statement}

Consider a rigid body equipped with a (3-axis) gyrometer and a (3-axis) accelerometer. The accelerometer is calibrated (in the sense of Section II-A), i.e. the matrices $A$ and $b$ in Equation (9) have already been identified, but the gyrometer is not. It is desired to calibrate the gyrometer in the frame of the accelerometer, i.e. such that, after this calibration, one can read the variable

$$
\Omega_{a c c}=R_{a c c} \Omega
$$

(the components of the vector rotation rates $\Omega$ in the accelerometer frame) without any misalignment, scale factor or bias. In other words, considering that, by default, one has

$$
\Omega_{g y r}=A_{0} \Omega_{a c c}+b_{0}
$$

it is desired to identify $A_{0}$ and $b_{0}$.

Proposition 1: Assuming that:

i) a properly calibrated accelerometer is available which delivers the measurements $\gamma_{a c c}$ and $\frac{d \gamma_{a c c}}{d t}$ in its own frame $\Re_{a c c}$. The readings satisfy $\gamma_{a c c}=R_{a c c} g$ where $R_{a c c}$ is the rotation from $\Re_{i}$ to $\Re_{a c c}, g$ is the gravity. (This is only true if the accelerometer is rotated with zero velocity, i.e. it is always on the rotation axis)

ii) the gyrometer to be calibrated gives the readings $\Omega_{g y r}$, then

- the readings from the accelerometer and from the gyrometer are related through the relation

$$
\frac{d \gamma_{a c c}}{d t}=-\Omega_{a c c} \times \gamma_{a c c}
$$

where $\Omega_{a c c}=R_{a c c} \Omega$

- the calibration matrix $A$ and bias $b$ such that

$$
\Omega_{a c c}=A \Omega_{g y r}+b
$$

can be estimated from the knowledge of $\Omega_{g y r}, \gamma_{a c c}$ and $\frac{d \gamma_{a c c}}{d t}$.

This proposition states that the gyroscopes can be calibrated in the accelerometer frame with a procedure which, in fact, does not require any expansive equipment and can be performed in-the-field by any operator. As will appear, the platform has simply to be rotated in every direction around the accelerometer. 
Proof: By making a cross product by $\gamma_{a c c}$ on the right side of Equation (12), and expanding the right-hand triple product according to the triple product expansion formula (a.k.a. Lagrange formula), one obtains

$$
\frac{d \gamma_{a c c}}{d t} \times \gamma_{a c c}=\left\|\gamma_{a c c}\right\|^{2} \Omega_{a c c}-\left(\Omega_{a c c}^{T} \gamma_{a c c}\right) \gamma_{a c c}
$$

$\Omega_{a c c}$ can be split into the sum of $\Omega_{a c c}^{\|}$parallel to $\gamma_{a c c}$, and $\Omega_{a c c}^{\perp}$ orthogonal to $\gamma_{a c c}$. Then, Equation (14) yields (in the plane orthogonal to $\gamma_{a c c}$ )

$$
\frac{d \gamma_{a c c}}{d t} \times \gamma_{a c c}=\left\|\gamma_{a c c}\right\|^{2} \Omega_{a c c}^{\perp}
$$

This means that, for a given sample, $\Omega_{a c c}^{\perp}$ can be determined from the knowledge of $\gamma_{a c c}$ and $\frac{d \gamma_{a c c}}{d t}$. In other words, $\Omega_{a c c}^{\perp}$ is observable from the measurement $\gamma_{a c c}$. According to the error model of the sensor given in Equation (13), this projection can be expressed in terms of the sought after calibration parameters $A$ and $b$ as

$$
\Omega_{a c c}^{\perp}=P_{B}\left(A \Omega_{g y r}+b\right)
$$

where $P_{B}$ is the projector on the plane orthogonal to $\gamma_{a c c}$.

Gathering Equation (15) and Equation (16) yields Equation (17) for a given set of measurements, say the $k^{t h}$.

By introducing the vectors $X_{k}$ depending only on the gyrometer measurement $\left(\Omega_{g y r}\right)_{k}$ and $Y_{k}$ depending only on the accelerometer measurements $\left(\gamma_{a c c}\right)_{k}$

$$
\begin{aligned}
X_{k} & =\left(\begin{array}{c}
\left(\Omega_{g y r}\right)_{k} \\
1
\end{array}\right) \\
Y_{k} & =\frac{\left(\frac{d \gamma_{a c c}}{d t}\right)_{k} \times\left(\gamma_{a c c}\right)_{k}}{\left\|\left(\gamma_{a c c}\right)_{k}\right\|}
\end{aligned}
$$

the equation can be simply rewritten under the linear form (in $A$ and $b$ )

$$
Y_{k}=\left(P_{B}\right)_{k}[A b] X_{k}
$$

Gathering a set of data (indexed by the $k$ variable), the parameters $A$ and $b$ can then be computed in a least square sense of the former equality. $A$ and $b$ are thus defined as the parameters minimizing the following cost function $J$

$$
J(\tilde{A}, \tilde{b})=\sum_{k=1}^{K}\left\|Y_{k}-\left(P_{B}\right)_{k}[\tilde{A} \tilde{b}] X_{k}\right\|^{2}
$$

Lemma 1: Denote $\left(E_{i}\right)_{i=1 . .12}$ a canonical base of $\mathbb{M}_{3,4}(\mathbb{R})$ with

$$
E_{1}=\left(\begin{array}{cccc}
1 & 0 & 0 & 0 \\
0 & 0 & 0 & 0 \\
0 & 0 & 0 & 0
\end{array}\right), E_{2}=\left(\begin{array}{cccc}
0 & 0 & 0 & 0 \\
1 & 0 & 0 & 0 \\
0 & 0 & 0 & 0
\end{array}\right), \ldots
$$

and use them to linearly decompose the two terms appearing in the sum (17)

with

$$
\mathbb{X}=\left[x_{i, j}\right]_{i=1 . .12, j=1.12}
$$

$$
\forall j, \sum_{k=1}^{K}\left(P_{B}\right)_{k} E_{j} X_{k} X_{k}^{T}=\sum_{i=1}^{12} x_{i, j} E_{i}
$$

$$
\mathbb{Y}=\left[y_{i}\right]_{i=1 . .12}
$$

with

$$
\sum_{k=1}^{K}\left(P_{B}\right)_{k} Y_{k}\left(X_{k}\right)^{T}=\sum_{i=1}^{12} y_{i} E_{i}
$$

Assuming $\mathbb{X}$ is invertible, the cost function $J(\tilde{A}, \tilde{b})$ has a unique minimum in $(A, b)$ defined by

$$
\left[\begin{array}{ll}
A & b
\end{array}\right]=\left(\begin{array}{llll}
m_{1} & m_{4} & m_{7} & m_{10} \\
m_{2} & m_{5} & m_{8} & m_{11} \\
m_{3} & m_{6} & m_{9} & m_{12}
\end{array}\right)
$$

with

$$
\left(\begin{array}{llll}
m_{1} & m_{2} & \ldots & m_{12}
\end{array}\right)^{T}=\mathbb{X}^{-1} \mathbb{Y}
$$

The proof of this lemma is proposed in Appendix I. It concludes the proof of Proposition 1 and gives an analytical expression (Equations (22) and (23)) of the sought after calibration matrix $A$ and bias $b$.

\section{PROPOSED OBSERVER}

The purpose of the discussed device consisting of distributed magnetometers and a low-cost IMU is to be used to obtain estimates of the velocity of the rigid body it is attached to. A first step is to reconstruct the attitude, which can be done, as previously discussed, using the IMU. A second step is to reconstruct the body velocity $v_{b}$ appearing in Equation (8). We now expose an algorithm to perform this estimation task in a general three-dimensional context. Consider Equation (8) and the following observer

$$
\left\{\begin{array}{l}
\dot{\hat{h}}=-\Omega \times \hat{h}+J\left[\hat{v}-L_{1}\left(\hat{h}-H_{b}\right)\right] \\
\dot{\hat{v}}=-\ell_{2} J^{T}\left(\hat{h}-H_{b}\right)
\end{array}\right.
$$

where $L_{1}$ is to be defined later. $H_{b}$ and $J$ are measurements from the sensors. The error dynamics is

$$
\left\{\begin{array}{l}
\dot{\tilde{h}}=\dot{\hat{h}}-\dot{H}_{b}=-\Omega \times \tilde{h}+J\left[\tilde{v}-L_{1}\left(\hat{h}-H_{b}\right)\right] \\
\dot{\tilde{v}}=\dot{\hat{v}}-\dot{v}_{b}=-\ell_{2} J^{T}\left(\hat{h}-H_{b}\right)
\end{array}\right.
$$

Proposition 2: The error dynamics, where $L_{1}=l_{1} J^{T}$, with $l_{1}>0$ and $l_{2}>0$ is globally asymptotically stable $\left(\lim _{t \rightarrow \infty} \tilde{h}=0, \lim _{t \rightarrow \infty} \tilde{v}=0\right)$ provided that $J$ is non singular.

Proof: Consider the candidate Lyapunov-function $W$

$$
W=\left\|\hat{h}-H_{b}\right\|^{2}+\frac{1}{\ell_{2}} \cdot\left\|\hat{v}-v_{b}\right\|^{2}
$$

Using (25), the time-derivative $\dot{W}$ is thus

$$
\begin{aligned}
\dot{W}= & -\left(\hat{h}-H_{b}\right)^{T} J L_{1}\left(\hat{h}-H_{b}\right)+\left(\hat{h}-H_{b}\right)^{T} J\left(\hat{v}-v_{b}\right) \\
& +\left(\hat{v}-v_{b}\right)^{T} J^{T}\left(\hat{h}-H_{b}\right)-\left(\hat{h}-H_{b}\right)^{T} L_{1}^{T} J^{T}\left(\hat{h}-H_{b}\right) \\
& -\left(\hat{v}-v_{b}\right)^{T} J^{T}\left(\hat{h}-H_{b}\right)-\left(\hat{h}-H_{b}\right)^{T} J\left(\hat{v}-v_{b}\right)
\end{aligned}
$$

Finally,

$$
\dot{W}=-\left(\hat{h}-H_{b}\right)^{T} \underbrace{\left[J L_{1}+L_{1}^{T} J^{T}\right]}_{Q}\left(\hat{h}-H_{b}\right)
$$




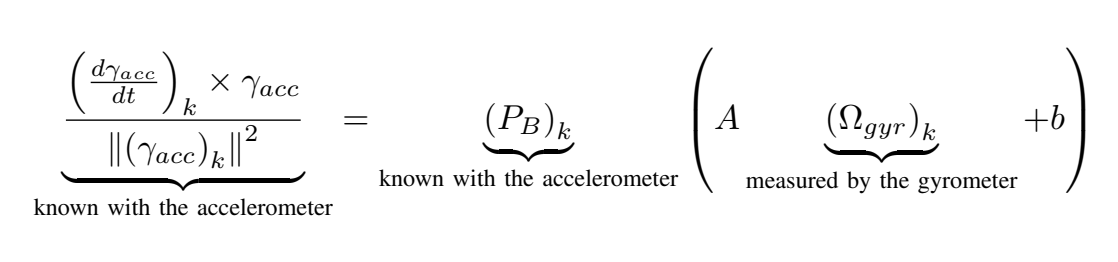

Choosing

$$
L_{1}=\ell_{1} J^{T}
$$

where the gain $\ell_{1}>0$ is scalar guarantees that $Q$ is positive semidefinite, thus $\dot{W} \leq 0$. If the set $\dot{W}\left(\hat{h}-H_{b}, \hat{v}-v_{b}\right)=0$ contains no other trajectory except the trivial one $\left(\hat{h}-H_{b}, \hat{v}-\right.$ $\left.v_{b}\right)=(0,0)$, then, from LaSalle's invariance principle [12], the origin is globally asymptotically stable.

Indeed, the invariant set is reduced to the origin. Trajectories lying in the set $\dot{W}=0$ are such that

$$
J^{T}\left(\hat{h}-H_{b}\right)=0
$$

Assuming $J$ has full rank, i.e. there are magnetic disturbances, we obtain

$$
\dot{\hat{h}}-\dot{H}_{b}=0
$$

However, $\dot{\hat{h}}-\dot{H}_{b}$ can be expressed as follows

$$
\dot{\hat{h}}-\dot{H}_{b}=J\left(\hat{v}-v_{b}\right)-\ell_{1} J J^{T}\left(\hat{h}-H_{b}\right)
$$

which yields

$$
0=\hat{v}-v_{b}
$$

To conclude, if the Jacobian $J$ is a full-rank matrix, and in practice it usually is due to the magnetic field disturbances, the observer reconstructs the value of the field $H_{b}$ and the velocity $v_{b}$ expressed in the body frame of coordinates $\Re_{b}$.

The final expression of the observer is

$$
\left\{\begin{array}{l}
\dot{\hat{h}}=-\Omega \times \hat{h}+J \hat{v}-\ell_{1} J J^{T}\left(\hat{h}-H_{b}\right) \\
\dot{\hat{v}}=-\ell_{2} J^{T}\left(\hat{h}-H_{b}\right)
\end{array}\right.
$$

where $\ell_{1}>0$ and $\ell_{2}>0$ are two scalar gains.

\section{EXPERIMENTAL RESULTS}

In this Section, our experimental testbench is presented. then, a 2D-trajectory of roughly $90 \mathrm{~m}$, which is reconstructed by integration of the velocity estimates provided by the observer, is reported. Finally, detailled results of velocity estimation obtained in a standard office building are compared against a reference velocity given by a laser telemeter.

\section{A. Experimental testbench and implementation}

Our testbench, pictured in Figure 1, consists of two separate modules connected by a communication wire also used to supply the sensors with electric power:

i) the sensing module comprising a wooden board and the various sensors.

ii) the data acquisition module where information from the various sensors are gathered by a microcontroller (namely an MPC555 from Motorola) and either sent to a computer via an RS232 link or stored in a SD card for post-treatment. Batteries for the whole system are also included in this module.

This design in two separated modules has been chosen to minimize magnetic noises around the sensors. From this perspective, it is important to realize that the microcontroller generates a small but non-negligible electromagnetic field. So, it should not be placed too close to the sensors. Moreover, the batteries, if put to close to the magnetometers, may act as a malicious screen occulting the magnetic field in a certain angle. All those possible sources of disturbances have been isolated in a remote second module, distinct from the sensing module.

The sensing module is placed on a wooden sledge to keep the movement in the plane. The distances between the sensors are precisely measured and further identified from experiments using a method described in [7].

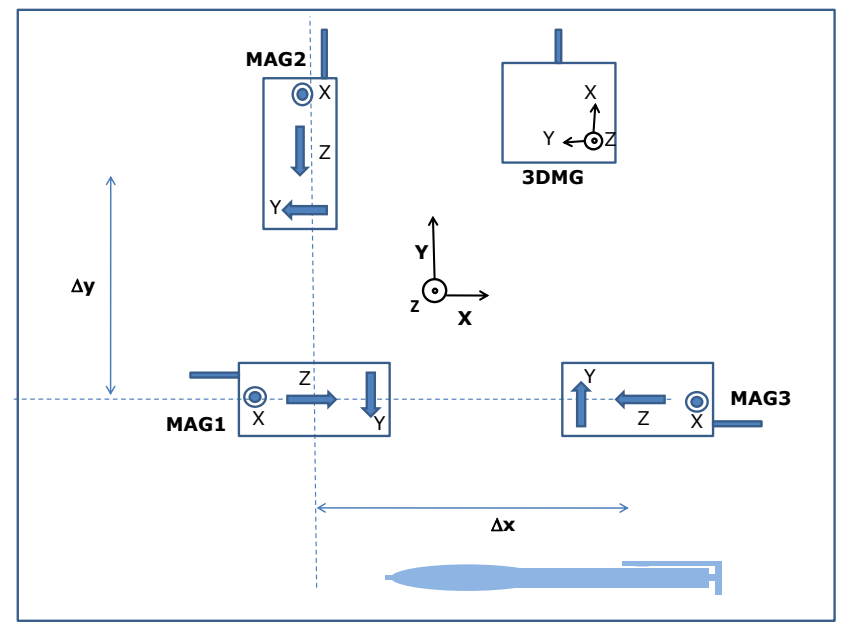

Fig. 1. Bredboard: schematic of the sensing module

1) Sensing module: Magnetometers

Numerous choices of magnetometers can be considered for this application. Three similar 3-axis fluxgate magnetometers FLC3-70 from Stefen Mayer Instrument are a good choice. Their range is \pm 2 gauss. A 24 bits $\mathrm{A} / \mathrm{D}$ converter for each axis and a small micro-controller which performs the communication have been integrated to the chip.

As discussed in Section II, measurements from these magnetometers are used to obtain two values expressed in the body frame: the value of the local magnetic field $H_{b}$, and the Jacobian of the magnetic field $J=J_{b}(H)$.

The Jacobian is computed from the measurements of the three magnetometers. Denote $M_{i}=\left(M_{i, x}, M_{i, y}\right)$ the values 
given by magnetometer $M_{i}$ (in the body frame). $M_{1}, M_{2}$, $M_{3}$ are located according to the schematic on Figure 1. A first approximation of the Jacobian is then

$$
J=\left(\begin{array}{cc}
\frac{M_{3, x}-M_{1, x}}{\Delta x} & \frac{M_{2, x}-M_{1, x}}{\Delta y} \\
\frac{M_{3, y}-M_{1, y}}{\Delta x} & \frac{M_{2, y}-M_{1, y}}{\Delta y}
\end{array}\right)
$$

where $\Delta_{x}$ is the distance between the sensors of magnetometers $M_{3}$ and $M_{1}$ which are both aligned on the $\mathrm{X}$-axis, and $\Delta_{y}$ the distance between the sensors of magnetometers $M_{2}$ and $M_{1}$ which are aligned on the Y-axis.

Accelerometer and gyrometer

The inertial measurement unit (IMU) 3DM-GX1 from Microstrain $^{\mathrm{TM}}$ gathers 3 angular rate gyroscopes, 3 singleaxis magnetometers, and 3 single-axis accelerometers, along with 16 bits $\mathrm{A} / \mathrm{D}$ converters and a micro-controller. In the presented setup, the IMU is mostly used for its gyroscope (iMEMS gyroscope from Analog Device), with a $30 \mathrm{~Hz}$ bandwidth. Its range is $\pm 300 \mathrm{deg} / \mathrm{s}$ and the in-run stability is $0.1 \mathrm{deg} / \mathrm{s}$. The accelerometers are only used in the auxiliary filter not presented in this paper which gives the heading in order to integrate the velocity. The unit costs nearly $1500 \$$ for $30 \mathrm{~g}$, and can perform measurements up to $76 \mathrm{~Hz}$ and send them via an RS232 serial link.

2) Data acquisition module: The aim of this module is to gather and store the information collected from all sensors. The acquisition technique proposed in [6] is implemented to account for timing discrepancies.

\section{B. Numerical scheme for data fusion algorithm implemen- tation}

The data fusion algorithm (24) is implemented on board a PC based system which solves differential equation in discrete time. Surprisingly enough, this discretization can reveal troublesome, and be the cause of unexpectedly misleading results.

Simple investigations reveal that usual explicit or implicit Euler numerical schemes are unable to reproduce pure rotational terms in the dynamics (24). For instance, a direct computation shows that, in the absence of any source term, while the analytical solution of

$$
\dot{H}=-\Omega \times H
$$

has a constant norm, solutions determined using Euler schemes do not and, rather, feature clearly wrong values of this norm. In the data fusion algorithm, this numerical effect is promptly interpreted as a fictitious variation of the magnetic field, wrongly attributed to a displacement of the rigid body.

To address this problem, we have realized that one can simply use a numerical scheme well-suited for oscillator (i.e. rotational) dynamics such as the ones developed in celestial mechanics studies. Our choice was to use a GaussJackson scheme (see e.g. [2], [3], [14]). An alternative is to consider higher order schemes as Adam's extrapolation formula (see [1]).

\section{Experimental results}

To illustrate the merits of the developed magneto-inertial method and to investigate its accuracy, two type of experiments have been conducted.

First, the bredboard pictured in Figure 1 was used indoors in corridors forming a rectangular path. The magnetic environment is impacted by disturbances typical of those commonly observed in office buildings. The rectangular path is realized with the bredboard fixed on a sledge to keep a 2D-trajectory. The heading used to integrate the estimated velocity in the inertial frame is given by an attitude filter not described here which makes use of the previously calibrated sensors on board (magnetometers, gyrometers and accelerometers). The results of the trajectory reconstruction using the velocity estimation technique are reported in Figure (2). This figure presents the reconstructed trajectory on the horizontal plane in order to compare it with a map of the building. One can see that the estimated distances are consistent with the map.

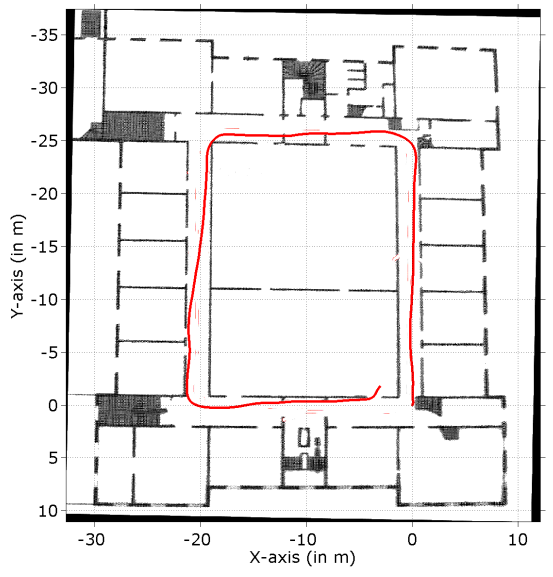

Fig. 2. 3D-Reconstructed trajectory projected on the map.

Second, the same bredboard is moved back and forth along a simple linear path in a corridor at the end of which a laser telemeter has been placed. This allows to precisely evaluate the representativeness of the velocity estimate, and of the subsequent relative position reconstruction. The corresponding data are represented in Figure (3).

One can clearly see the very good quality of the velocity estimate, which, in particular, kindly reproduces the sharp accelerations. However, some discrepancies between the estimated velocity and the laser reconstructed velocity are still visible in this figure. The estimated velocity is also very noisy. These problems are currently under investigation. In particular, using in the observer, not only the magnetic gradients, but also their second and third order derivatives seems to have already limited the noise and improved high dynamic movement reconstruction. Further development in that direction are under consideration. 


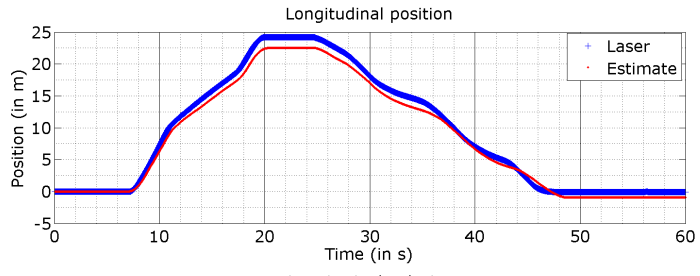

Longitutinal velocity

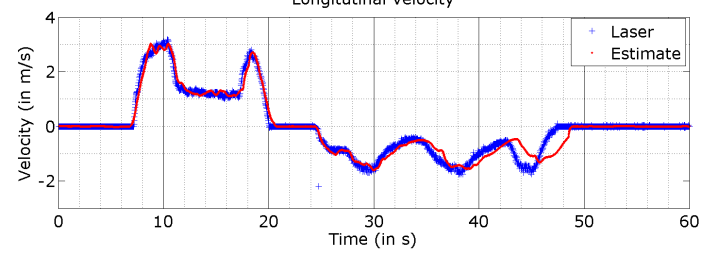

Fig. 3. Velocity (and integrated position) in a long corridor. The velocity estimated by the filter is compared against a laser reference.

\section{CONCLUSION AND FUTURE DIRECTIONS}

In this paper, we have shown that the information lying in the disturbances of the magnetic field is not only interesting to improve the accuracy of a purely inertial approach as shown in [17], [16], but also reliable enough to provide an accurate velocity estimate without accelerometer. An observer for the velocity based only on measurements from a set of distributed magnetometers (and a gyroscope) has been proposed and its convergence to the actual velocity in the body frame has been proved. To improve the attitude estimation, reconciliating data from the accelerometer and the gyroscope, through a calibration technique, has been a subject of particular interest, for which we have proposed a practical method. The robustness of the sensing and data fusion system can certainly be improved further. This is a subject of future development. Experiments with 3Dtrajectories will also be conducted soon.

\section{ACKNOWLEDGMENTS}

This work was partially funded by the SYSTEM@TIC PARIS-REGION Cluster in the frame of project LOCINDOOR.

\section{REFERENCES}

[1] M. Abramowitz and I. Stegun. Handbook of Mathematical Functions. Dover, New York, 1965.

[2] R. R. Bate, D. D. Mueller, and J. E. White. Fundamentals of Astrodynamics, chapter 9.6.4 The Gauss-Jackson method, pages 414419. Dover Publications Inc., 1971.

[3] M. M. Berry and L. M. Healy. Implementation of gauss-jackson integration for orbit propagation. The Journal of the Astronautical Sciences, 52(3):331-357, July-September 2004.

[4] A. B. Chatfield. Fundamentals of High Accuracy Inertial Navigation, volume 174 of Progress in Astronautics and Aeronautics Series. AIAA, 1997.

[5] E. Dorveaux, D. Vissière, A.-P. Martin, and N. Petit. Iterative calibration method for inertial and magnetic sensors. pages $8296-$ 8303, dec. 2009.
[6] E. Dorveaux, D. Vissière, A.-P. Martin, and N. Petit. Time-stamping for an array of low-cost sensors. 2009.

[7] E. Dorveaux, D. Vissière, and N. Petit. On-the-field calibration of an array of sensors. 2010.

[8] P. Faurre. Navigation inertielle et filtrage stochastique. Méthodes mathématiques de l'informatique. Dunod, 1971.

[9] W. T. Fong, S. K. Ong, and A. Y. C. Nee. Methods for in-field user calibration of an inertial measurement unit without external equipment. Measurement Science and Technology, 19(8):085202-+, Aug. 2008.

[10] M. S. Grewal, L. R. Weill, and A. P. Andrews. Global positioning systems, inertial navigation, and integration. Wiley Inter-science, 2001.

[11] T. Hamel and R. Mahony. Attitude estimation on so[3] based on direct inertial measurements. In Proc. of the IEEE International Conference on Robotics and Automation (ICRA), 2006.

[12] H. K. Khalil. Nonlinear Systems. MacMillan, 1992.

[13] L. Ojeda and J. Borenstein. Non-GPS navigation for security personnel and first responders. Journal of Navigation, 60(3):391-407, September 2007.

[14] A. Sharif. GPS satellite orbit integration by the Gauss-Jackson process. Buletin Ukur, 3:35-48, 1992.

[15] I. Skog, J.-O. Nilsson, and P. Händel. Evaluation of zero-velocity detectors for foot-mounted inertial navigation systems. In Proc. of the $1^{\text {st }}$ Indoor Positioning and Indoor Navigation Conference (IPIN), 2010.

[16] D. Vissière, A. P. Martin, and N. Petit. Using magnetic disturbances to improve IMU-based position estimation. 2007.

[17] D. Vissière, A.-P. Martin, and N. Petit. Using spatially distributed magnetometers to increase IMU-based velocity estimation in perturbed areas. 2007.

[18] U. Walder, T. Bernoulli, G. Glanzer, and T. WieSSflecker. Contextadaptive algorithms to improve indoor positioning with inertial sensors. In Proc of the 1st Indoor Positioning Indoor Navigation (IPIN) Conference, 2010.

\section{APPENDIX I}

\section{Proof of Lemma 1}

To minimize the convex function (21), its derivative is required. The following lemma is useful.

Lemma 2: Denote $y \in \mathbb{R}^{m}, x \in \mathbb{R}^{n}$ and $S \in \mathbb{S}_{n}(\mathbb{R})$ a skew symmetric matrix. Define $f: M \in \mathbb{M}_{m, n}(\mathbb{R}) \longrightarrow$ $\|y-S M x\|^{2}$. Then, $f$ is differentiable and its derivative is

$$
D f(M)=\left[\frac{\partial f}{\partial M_{i, j}}\right]_{\substack{i=1 . . m \\ j=1 . . n}}=-2 \cdot S(y-S M x) x^{T}
$$

Proof: First, one shail expand the expression of $f$,

$$
\begin{aligned}
f(M)= & \\
& \sum_{i}\left(y_{i}-\sum_{j, k} S_{i, j} M_{j, k} x_{k}\right)\left(y_{i}-\sum_{p, q} S_{i, p} M_{p, q} x_{q}\right) \\
= & \sum_{i} y_{i}^{2}-2 \cdot \sum_{i, j, k} y_{i} S_{i, j} M_{j, k} x_{k}+ \\
& \sum_{i, j, k, p, q} x_{k}^{T} M_{j, k} S_{i, j} S_{i, p} M_{p, q} x_{q}
\end{aligned}
$$

Then, for any $(u, v) \in[1, m] \times[1, n]$, the derivative of $f(M)$ with respect to any component $M_{u, v}$ is

$$
\begin{aligned}
\frac{\partial f}{\partial M_{u, v}}(M)= & -2 \cdot \sum_{i} y_{i} S_{i, u} x_{v}+\sum_{i, p, q} x_{v} S_{i, u} S_{i, p} M_{p, q} s_{q} \\
& +\sum_{i, j, k} x_{k} M_{j, k} S_{i, j} S_{i, u} x_{v}
\end{aligned}
$$


Because $\mathrm{S}$ is skew symmetric,

$$
\begin{aligned}
\frac{\partial f}{\partial M_{u, v}}(M)= & -2 \cdot\left(\sum_{i} S_{u, i} y_{i}\right) x_{v} \\
& +2\left(\sum_{i, p, q} S_{u, i} S_{i, p} M_{p, q} x_{q}\right) x_{v} \\
= & -2 \cdot(S y)_{u} x_{v}+2 \cdot\left(S^{2} M x\right)_{u} x_{v} \\
= & -2 \cdot(S(y-S M x))_{u} x_{v}
\end{aligned}
$$

which yields

$$
\begin{aligned}
D_{f}(M) & =\left[\frac{\partial f}{\partial M_{u, v}}\right]_{u=1 . . m, v=1 . . n} \\
& =-2 \cdot S(y-S M x) x^{T}
\end{aligned}
$$

$J(\tilde{A}, \tilde{B})$ is a convex and differentiable function. It thus reaches its minimum when its derivative is zero. As $\left(P_{B}\right)_{k}$ is an orthogonal projector, its matrix is skew symmetric. Lemma 2 applies, leading to

$$
\begin{aligned}
0 & =-2 \cdot \sum_{k=1}^{K}\left(P_{B}\right)_{k}\left(Y_{k}-\left(P_{B}\right)_{k}[A b] X_{k}\right)\left(X_{k}\right)^{T} \\
\sum_{k=1}^{K} Y_{k}\left(X_{k}\right)^{T} & =\sum_{k=1}^{K}\left(P_{B}\right)_{k}[A b] X_{k} X_{k}^{T}
\end{aligned}
$$

Using the canonical base $\left(E_{i}\right)_{i=1 . .12}$ of $\mathbb{M}_{3,4}(\mathbb{R})$ defined in Lemma 1 , and writing $[A b]$ under the form

$$
\left[\begin{array}{ll}
A & b
\end{array}\right]=\left(\begin{array}{llll}
m_{1} & m_{4} & m_{7} & m_{10} \\
m_{2} & m_{5} & m_{8} & m_{11} \\
m_{3} & m_{6} & m_{9} & m_{12}
\end{array}\right)=\sum_{j=1}^{12} m_{j} E_{j}
$$

The relation (31) can be rewritten as

$$
\underbrace{\sum_{k=1}^{K} Y_{k} X_{k}^{T}}_{\sum_{i=1}^{12} y_{i} E_{i}}=\sum_{j=1}^{12} m_{j} \cdot \underbrace{\left(\sum_{k=1}^{K}\left(P_{B}\right)_{k} E_{j} X_{k} X_{k}^{T}\right)}_{\sum_{j=1}^{12} x_{i, j} E_{i}}
$$

This yields

$$
\begin{aligned}
y_{i} & =\sum_{j=1}^{12} x_{i, j} m_{j} \forall i=1 . .12 \\
\mathbb{Y} & =\mathbb{X}\left(\begin{array}{llll}
m_{1} & m_{2} & \ldots & m_{12}
\end{array}\right)^{T}
\end{aligned}
$$

Under the assumption that $\mathbb{X}$ is invertible, one obtains

$$
\left(\begin{array}{llll}
m_{1} & m_{2} & \ldots & m_{12}
\end{array}\right)^{T}=\mathbb{X}^{-1} \mathbb{Y}
$$

which concludes the proof.

\section{APPENDIX II}

From definition (2),

$$
H_{b}=R H_{i}
$$

then

$$
\frac{d H_{b}}{d t}=\frac{d R}{d t} H_{i}+R \frac{d H_{i}}{d t}
$$

$R$ being orthogonal, one has

$$
R R^{T}=R^{T} R=I
$$

and, one has

$$
\frac{d R}{d t}=-[\Omega] R
$$

where $[\Omega]$ is the matrix associated with the left vector cross product by $\Omega$

$$
[\Omega] u=\Omega \times u
$$

Then, one obtains, with (3)

$$
\begin{aligned}
\frac{H_{b}}{d t} & =-\Omega \times H_{b}+R J_{i}(H) v_{i} \\
& =-\Omega \times H_{b}+J_{b}(H) v_{b}
\end{aligned}
$$

which yields Equation (4). 